\title{
Persistent C-peptide secretion in Type 1 diabetes and its relationship to the genetic architecture of diabetes
}

\author{
Paul M. McKeigue ${ }^{\text {* }}$ (D), Athina Spiliopoulou' ${ }^{1}$ Stuart McGurnaghan², Marco Colombo ${ }^{1}$, Luke Blackbourn², \\ Timothy J. McDonald ${ }^{9}$, Suna Onengut-Gomuscu ${ }^{8}$, Stephen S. Rich ${ }^{8}$, Colin N. A. Palmer ${ }^{10}$, \\ John A. McKnight ${ }^{5}$, Mark W. J. Strachan ${ }^{5}$, Alan W. Patrick ${ }^{4}$, John Chalmers ${ }^{6}$, Robert S. Lindsay ${ }^{3}$, \\ John R. Petrie ${ }^{3}$, Sandeep Thekkepat ${ }^{7}$, Andrew Collier ${ }^{11}$, Sandra MacRury ${ }^{12}$ and Helen M. Colhoun ${ }^{2}$
}

\begin{abstract}
Background: The objective of this cross-sectional study was to explore the relationship of detectable C-peptide secretion in type 1 diabetes to clinical features and to the genetic architecture of diabetes.

Methods: C-peptide was measured in an untimed serum sample in the SDRNT1BIO cohort of 6076 Scottish people with clinically diagnosed type 1 diabetes or latent autoimmune diabetes of adulthood. Risk scores at loci previously associated with type 1 and type 2 diabetes were calculated from publicly available summary statistics.

Results: Prevalence of detectable C-peptide varied from 19\% in those with onset before age 15 and duration greater than 15 years to $92 \%$ in those with onset after age 35 and duration less than 5 years. Twenty-nine percent of variance in C-peptide levels was accounted for by associations with male gender, late age at onset and short duration. The SNP heritability of residual C-peptide secretion adjusted for gender, age at onset and duration was estimated as 26\%. Genotypic risk score for type 1 diabetes was inversely associated with detectable C-peptide secretion: the most strongly associated loci were the HLA and INS gene regions. A risk score for type 1 diabetes based on the HLA DR3 and DQ8-DR4 serotypes was strongly associated with early age at onset and inversely associated with C-peptide persistence. For C-peptide but not age at onset, there were strong associations with risk scores for type 1 and type 2 diabetes that were based on SNPS in the HLA region but not accounted for by HLA serotype.

Conclusions: Persistence of C-peptide secretion varies widely in people clinically diagnosed as type 1 diabetes. C-peptide persistence is influenced by variants in the HLA region that are different from those determining risk of early-onset type 1 diabetes. Known risk loci for diabetes account for only a small proportion of the genetic effects on C-peptide persistence.
\end{abstract}

Keywords: C-Peptide, Diabetes mellitus type 1, Age at diagnosis, Insulin secretion, Genetics, Cross-sectional studies

\section{Background}

Studies using sensitive assays for C-peptide have shown that some degree of residual insulin secretion commonly persists for more than 5 years after diagnosis of type 1 diabetes [1-4]. However, few studies have examined the frequency with which $\mathrm{C}$-peptide secretion persists after

\footnotetext{
*Correspondence: paul.mckeigue@ed.ac.uk

'Usher Institute of Population Health and Informatics, University of Edinburgh, Old Medical School, Teviot Place, Edinburgh EH8 9AG, UK

Full list of author information is available at the end of the article
}

long duration of type 1 diabetes or across a wide range of ages of onset. Using an assay with lower limit of detection $17 \mathrm{pmol} / \mathrm{l}$, the prevalence of detectable C-peptide in nonfasting serum samples was reported to be much lower $(6 \%$ versus $78 \%$ ) in those diagnosed in childhood with long ( $>40$ years) duration than in those diagnosed as adults with short duration (3-5 years) [3]. In another study, fasting C-peptide at diagnosis was found to be lower and to decline more steeply with time in those with younger age at onset [5].

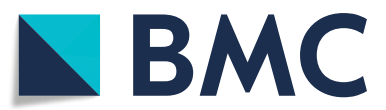

(C) The Author(s). 2019 Open Access This article is distributed under the terms of the Creative Commons Attribution 4.0 International License (http://creativecommons.org/licenses/by/4.0/), which permits unrestricted use, distribution, and reproduction in any medium, provided you give appropriate credit to the original author(s) and the source, provide a link to the Creative Commons license, and indicate if changes were made. The Creative Commons Public Domain Dedication waiver (http://creativecommons.org/publicdomain/zero/1.0/) applies to the data made available in this article, unless otherwise stated. 
Nonetheless at any age of onset or duration, there is variation in C-peptide persistence, the determinants of which are poorly understood but may be partly genetically determined. One recent genome-wide association study in 3479 people with type 1 diabetes, but mostly diagnosed in childhood, identified a locus on chromosome 1 and multiple variants in the HLA region associated with C-peptide levels adjusted for sex, age at diagnosis and diabetes duration [6].

The objective of this study was to investigate the relationship of detectable C-peptide secretion to clinical features and to the genetic architecture of diabetes in a population-based cohort of people with a clinical diagnosis of type 1 diabetes spanning a wide range of age at diagnosis and duration. A specific objective was to test whether heterogeneity in C-peptide persistence in people clinically diagnosed as type 1 was explained by inclusion of misdiagnosed cases of type 2 diabetes or by genetic heterogeneity in cases of type 2 diabetes.

\section{Methods}

\section{Study population}

The Scottish Diabetes Research Network Type 1 Bioresource (SDRNT1BIO) is a cohort of people clinically diagnosed as type 1 diabetes aged 16 years and older at recruitment. Questionnaire data and samples obtained on the day of recruitment were linked to clinical data from the Scottish Care Information Diabetes Collaboration electronic health record [7]. The cohort comprises one third of the adult population with type 1 diabetes in Scotland and its representativeness has been described in detail [8]. In Scotland, most people diagnosed with diabetes do not have auto-antibodies measured at diagnosis, and the clinical diagnosis of type 1 is based on age at diagnosis, time to insulin, any history of ketoacidosis, and exclusion of monogenic subtypes of diabetes.

Of 6127 people recruited into the study, there were 6076 with a clinical diagnosis of type 1 diabetes or latent autoimmune diabetes of adulthood after excluding those diagnosed with monogenic subtypes of diabetes (intentionally recruited for the cohort) or diabetes from other causes. Median age at onset was 21 (interquartile range 12 to 31) years, and median duration of diabetes at enrolment was 21 (interquartile range 11 to 31 ) years. In 120 of these individuals, more than 1 year had elapsed from diagnosis to starting insulin, ascertained from prescription records and questionnaire responses.

\section{Laboratory measurements}

Non-fasting serum samples were obtained at clinic visit in 5928 of those clinically diagnosed as type 1 . The median time from sampling to freezing at $-80^{\circ} \mathrm{C}$ was $2 \mathrm{~h} 15 \mathrm{~min}$ (interquartile range $1 \mathrm{~h} 30 \mathrm{~min}$ to $3 \mathrm{~h} 10 \mathrm{~min}$ ). Plasma glucose measured in these blood samples was greater than $5 \mathrm{mmol} / \mathrm{l}$ in $88 \%$ of individuals. Non-fasting random $\mathrm{C}$-peptide levels in people with type 1 diabetes are highly correlated with C-peptide levels after a mixed meal [9]. C-peptide measurements on these samples were undertaken at the Exeter Clinical Laboratory using the Roche electrochemiluminescence assay [10], with a lower limit of detection of C-peptide of $3 \mathrm{pmol} / \mathrm{l}$. Autoantibodies to glutamic acid decarboxylase (GAD65), tyrosine phosphorylase-related protein 2 (IA2) and zinc transporter 8 (ZnT8) were measured at the Exeter laboratory, which participates in the Diabetes Antibody Standardisation Programme [11].

Antibody titres exceeding the 97.5th percentile of the reference range were scored as positive. The 97.5th percentiles for GAD and IA2 are 11 and 7.5 World Health Organization (WHO) units/ml respectively. For ZnT8, the 97.5th percentile was $65 \mathrm{WHO}$ units $/ \mathrm{ml}$ in those aged up to age 30 years and 9.1 in those aged more than 30 years. Those with at least one antibody level above the reference range were classified as autoantibody-positive. These autoantibody measurements were used in combination with C-peptide measurements used to identify possible misdiagnosed cases of type 2 diabetes. The rationale for this was that in those who have residual beta-cell function as indicated by high C-peptide levels, we would expect autoantibodies to be still present if diabetes were caused by autoimmmune beta cell damage. This classification based on C-peptide levels and autoantibody status was validated by examining genotypic scores as described in the "Results" section.

\section{Genotyping}

The cohort was typed with the Illumina Human Core Exome 241.0 chip at the Center for Public Health Genomics, University of Virginia. After qualitychecks, genotypes were available on $r$ of those clinically diagnosed as type 1 diabetes. Genotypes were phased and imputed to the UK10K reference panel with the EAGLE algorithm [12], and the imputed genotypes were filtered to exclude SNPs with minor allele frequency less than 0.02 or proportion of information extracted less than 0.7 .

\section{Calculation of genotypic scores from summary statistics}

Genotypic risk scores for type 1 diabetes and type 2 diabetes were computed using the GENOSCORES platform described elsewhere [13]. Univariate regression coefficients from publicly available meta-analyses $[14,15]$ were supplemented with single-SNP scores for additional type 1 diabetes-associated loci reported in a further metaanalysis from which only one SNP per locus was published [16]. Other meta-analyses that did not give the magnitudes and signs of the effects could not be used to calculate scores. Diabetes-associated SNPs were filtered at a threshold $p$ value of $10^{-5}$. Locus-specific scores were generated 
for regions containing at least one SNP with $p$ value less than $10^{-6}$ and separated from other filtered SNPs by a gap of at least one megabase. All other filtered SNPs were combined into a residual genome-wide score. The threshold $p$ value used to designate a genomic region as a diabetes-associated locus limits the number of regions thus designated but does not make any difference to the genome-wide score.

The GENOSCORES platform adjusts the locus-specific scores for linkage disequilibrium between SNP genotypes by premultiplying the vector of univariate SNP coefficients, obtained from summary GWAS results, by the generalized inverse of the correlation matrix between these genotypes. This correlation matrix was estimated from the 1000 Genomes European ancestry reference panel. The relative weights of the SNPs obtained by this procedure approximate the weights that would be obtained by fitting a multivariate regression model to the individual-level data. In principle, this method should capture additive effects across each genomic region, but not interaction effects between alleles at the same locus (dominance) or different loci (epistasis).

After restricting to SNPs that were contained in the type 1 Bioresource genotype dataset, this procedure generated 41 locus-specific scores for type 1 diabetes and 60 locus-specific scores for type 2 diabetes. There were five risk loci that were common to both types of diabetes-BMP8A, HLA region, $C E N P W, A S C C 2$ and $B C A R 1 / C T R B 1 / C T R B 2$. Although the HLA region is not generally considered an established risk locus for type 2, it was included as a locus-specific score based on the criterion of at least one SNP with $p$ value less than $10^{-6}$. For each diabetes type, locusspecific scores and the residual genome-wide score were summed over loci to obtain the full genome-wide score.

For type 1 diabetes, separate scores were constructed for HLA serotypes and for other SNPs in the HLA region. HLA serotypes at the DQB1 and DRB1 loci were imputed from the untyped SNPs using the HIBAG program [17] with reference serotypes based on all European ancestry individuals in the 1000 Genomes panel [18]. Alleles at these loci were grouped as follows: 0301 to 0304 at the DRB1 locus as DR3, 0401 to 0413 at the DRB1 locus as DR4 and 0302 to 0305 at the DQB1 locus as DQ8. Serotypes at these two loci were classified into six groups-DR3/DR4-DQ8, DR3/DR3, DR4-DQ8/DR4DQ8, DR4-DQ8/X, DR3/X and $\mathrm{X} / \mathrm{X}$ - to which score weights were assigned as published by Oram et al. [19]. The HLA region-specific polygenic score for type 1 diabetes was regressed on this HLA serotype score, and the residuals from this regression were included in the analysis as the "HLA residual" score. The HLA region was excluded from the genome-wide score for type 2 , so that the type 2 score could be used to discriminate between liability to type 2 and liability to type 1 .

Each locus-specific score was scaled to unit standard deviation so that effect sizes could be compared. The genome-wide genotypic scores were standardized to have zero mean and unit standard deviation in White British participants without diabetes in UK Biobank.

\section{Comparison with genotypic scores in UK Biobank participants}

To validate the classification of diabetes type in the SDRNT1BIO cohort, we compared the distributions of genotypic scores in these groups with the genotypic scores in UK Biobank participants with and without diabetes whose self-reported ethnicity was White British. Of these participants, 16,427 reported that they had been diagnosed with diabetes (excluding those diagnosed only with gestational diabetes). One thousand four hundred thirteen of these were categorized as type 1 diabetes based on questionnaire report that they had been diagnosed before age 50 years and started insulin within a year of diagnosis, and the remaining 15,014 were categorized as type 2 diabetes.

\section{Statistical analysis}

For modelling associations of clinical covariates, age at onset was transformed by taking the square root, and $\mathrm{C}$-peptide levels were transformed by taking the logarithm to base 10, and setting the log transform of values below detection threshold to zero. As preliminary analysis showed that these associations varied with age at onset, interaction terms with age at onset were included in these models.

To allow for relatedness, the relationship matrix was computed from the unimputed genotypes and the $\mathrm{R}$ package GMMAT [20] was used to fit linear mixed models for age at onset and for C-peptide. The model for $\mathrm{C}$ peptide was adjusted for sex, age at onset and duration. Fitting this linear mixed model yields an estimate of heritability and allows genome-wide SNP association tests to be computed efficiently from the gradient of the loglikelihood (efficient score) at the null.

\section{Results}

Relation of C-peptide secretion to age at onset, duration and auto-antibody status

Table 1 shows the frequency of detectable C-peptide by age at onset and duration among those classified clinically as type 1 diabetes. Prevalence of detectable C-peptide levels varied from 19\% in those with onset before age 15 and duration greater than 35 years to $72 \%$ in those with onset after age 35 and duration less than 15 years. For C-peptide levels above $50 \mathrm{pmol} / \mathrm{l}$-a threshold previously associated with better glycaemic control [21] - the 
Table 1 Prevalence of detectable C-peptide by age at onset and duration (years)

\begin{tabular}{|c|c|c|c|c|c|}
\hline Age at onset & 0 to 15 & 15 to 25 & 25 to 35 & $35-$ & \\
\hline \multicolumn{6}{|l|}{ Duration } \\
\hline 0 to 5 & $13 / 17$ & $96 \% \quad 178 / 186$ & $94 \% \quad 161 / 172$ & $92 \%$ & $262 / 285$ \\
\hline 5 to 10 & $79 / 128$ & $67 \% \quad 110 / 165$ & $78 \% \quad 107 / 138$ & $74 \%$ & $188 / 253$ \\
\hline 10 to 15 & $60 / 237$ & $55 \% \quad 89 / 163$ & $52 \% \quad 103 / 197$ & $57 \%$ & $129 / 225$ \\
\hline 15 or more & $19 \% 317 / 1643$ & $26 \% \quad 259 / 996$ & $36 \% \quad 253 / 705$ & $42 \%$ & $174 / 418$ \\
\hline All & $23 \% \quad 469 / 2025$ & $42 \% \quad 636 / 1510$ & $51 \% 624 / 1212$ & $64 \%$ & $753 / 118$ \\
\hline
\end{tabular}

prevalence rates in the same groups were respectively $4 \%$ and $58 \%$. Geometric mean C-peptide levels by age at onset and duration (smoothed by LOESS regression) are shown in Fig. 1.

Table 2 shows that most people who had C-peptide levels above $600 \mathrm{pmol} / \mathrm{l}$ and had been diagnosed at least 5 years earlier were autoantibody-negative. Accordingly, the 203 individuals with C-peptide greater than $600 \mathrm{pmol} / \mathrm{l}$ who were negative for all three autoantibodies were classified as "possible type 2", and all others as "definite type 1 ".

To examine whether the genetic risk profile of the possible type 2 individuals was more similar to that of people with type 2 than type 1 diabetes, we plotted the contours of the joint probability distribution of the genome-wide risk scores for type 1 and type 2 diabetes in those classified as definite type 1 diabetes (Fig. 2). The mean of the type 1 genotypic risk score in the cohort is about 1.6 standard deviations above the mean for White British UK Biobank participants without diabetes. The mean genotypic risk scores for type 1 and type 2 in the cohort members classified as possible type 2 were close to the mean for UK Biobank participants classified as type 2. On this basis, those classified as possible type 2 were excluded from the further analyses reported below.

\section{Heritability and associations with clinical covariates and genome-wide genotypic risk scores Age at onset}

The SNP heritability of age at onset (transformed to the square root and adjusted for gender) was estimated as 0.3. Age at onset was associated inversely with genotypic risk score for type 1 diabetes (standardized regression coefficient $-0.23 \mathrm{yr}^{0.5}, p=10^{-50}$ ). However, gender and genotypic risk scores accounted for only $4 \%$ of the variance of age at onset.

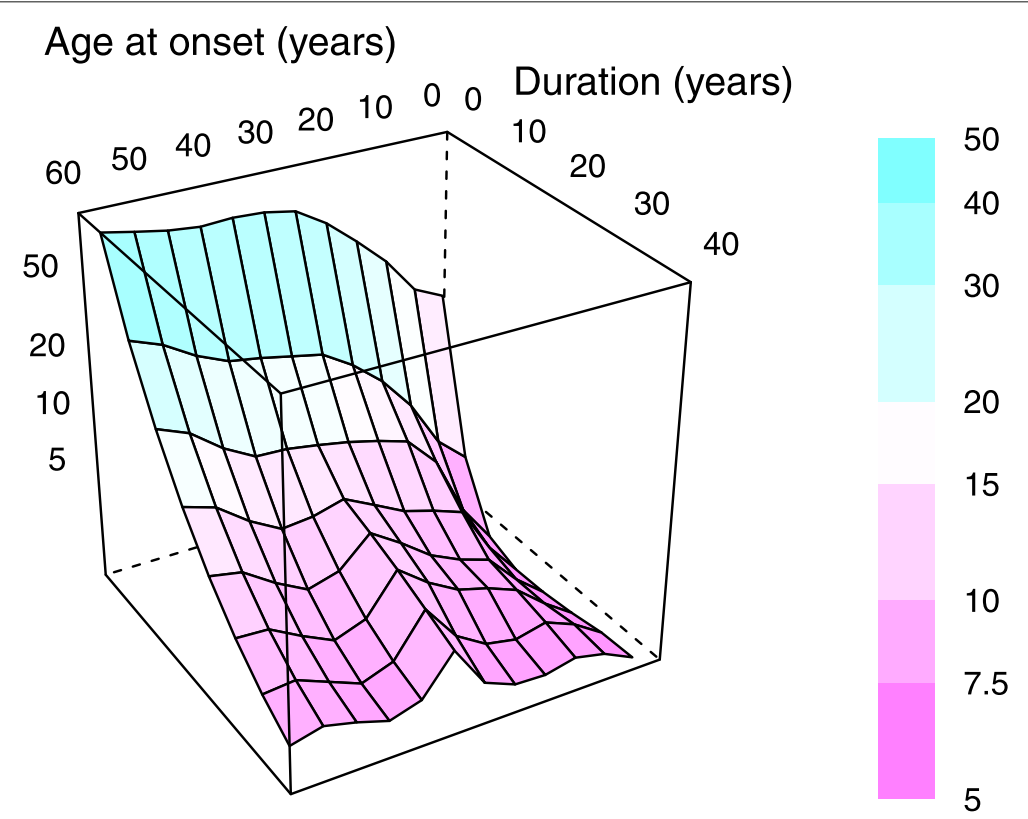

Fig. 1 Geometric mean plasma C-peptide (pmol/l) by age at onset and duration. A smoothed fit of log C-peptide to age at onset and duration was computed by LOESS regression of polynomial degree 2 and span 0.25 , then evaluated over a grid of values of the predictor variables. The C-peptide level (pmol/l) of each panel is encoded both as its colour and as its coordinate on the vertical axis 
Table 2 Proportion negative for autoantibodies by duration (years) and C-peptide (pmol/l)

\begin{tabular}{|c|c|c|c|c|}
\hline C-peptide range & {$[0,30]$} & $(30,200]$ & $(200,600]$ & $(600,7 e+03]$ \\
\hline \multicolumn{5}{|l|}{ Duration } \\
\hline 0 to 5 & $5 \% \quad 5 / 92$ & $9 \% \quad 15 / 168$ & $12 \% \quad 28 / 243$ & $35 \% \quad 54 / 155$ \\
\hline 5 to 10 & $15 \% \quad 49 / 326$ & $13 \% \quad 26 / 196$ & $24 / 87$ & $47 / 68$ \\
\hline 10 to 15 & $17 \% \quad 97 / 568$ & $21 \% \quad 26 / 122$ & $25 / 56$ & $53 / 71$ \\
\hline 15 or more & $30 \% 997 / 3272$ & $36 \% \quad 108 / 300$ & $59 \% \quad 68 / 116$ & $80 \% \quad 49 / 61$ \\
\hline All & $27 \% 1148 / 4258$ & $22 \% \quad 175 / 786$ & $29 \% \quad 145 / 502$ & $57 \% \quad 203 / 355$ \\
\hline
\end{tabular}

\section{C-peptide}

The heritability of C-peptide (log-transformed and adjusted for gender and age at onset $\times$ duration) was estimated as 0.26 . In a linear regression model with $\log$ $\mathrm{C}$-peptide as dependent variable, $29 \%$ of variance in $\log$ $\mathrm{C}$-peptide was explained by age at onset, duration and gender. As early age at onset was associated in this cohort with female sex (coefficient with square root of age as dependent variable -0.17 ), some of the association of female sex with lower $C$-peptide levels was explained by age at onset.

Table 3 summarizes a regression model with C-peptide as dependent variable and plasma glucose (dichotomized at $5 \mathrm{mmol} / \mathrm{l})$, age at onset, gender, duration, genotypic risk scores and body mass index as covariates. Terms for interaction of age at onset with other covariates are included in this model. To make the results easier to interpret, covariates are centred to have zero mean so that each main effect represents the predicted effect of that variable when other covariates are at their mean values. C-peptide levels were not associated with plasma glucose levels. C-peptide levels were associated positively with age at onset and higher genotypic score for type 2 diabetes and inversely with female gender, duration and genotypic score for type 1 diabetes (Table 3 ). The effects of gender, duration and body mass index were dependent upon age at onset, and the signs of the coefficients (main effect and interaction effect in the same direction) show that these effects were stronger in late-onset than in early-onset cases.

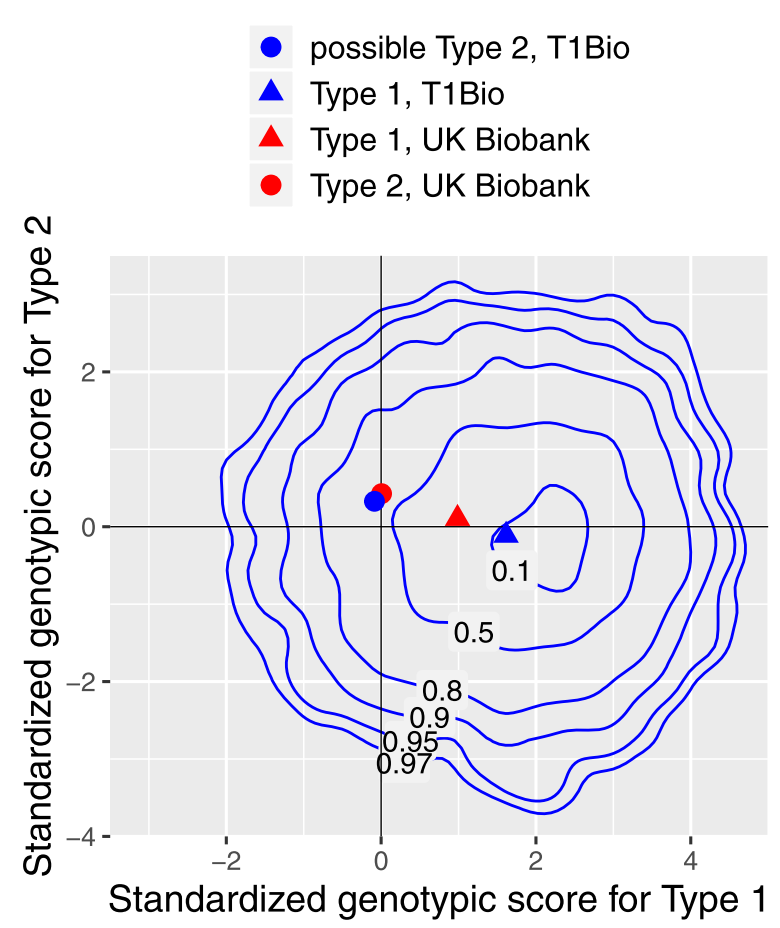

Fig. 2 Joint probability contours of genotypic scores for type 1 and type 2 diabetes excluding those classified as possible type 2. Labels show probability enclosed by each contour. Scores standardized to zero mean and unit standard deviation in UK Biobank White British participants without diabetes 
Table 3 Regression of log C-peptide on gender, age at onset, duration, glucose, body mass index and genotypic risk scores for diabetes, excluding those with possible type 2 defined by autoantibody status and C-peptide level

\begin{tabular}{llll}
\hline & Estimate & Std. error & $p$ value \\
\hline Plasma glucose $>5 \mathrm{mmol} / \mathrm{l}$ & 0.0066 & 0.029 & 0.8 \\
$\sqrt{\text { age onset }}$ & 0.11 & 0.0083 & $2 \mathrm{e}-40$ \\
Female gender & -0.074 & 0.022 & 0.001 \\
Duration (years) & -0.03 & 0.00093 & $2 \mathrm{e}-206$ \\
Type 1 DM risk score & -0.04 & 0.0088 & $5 \mathrm{e}-06$ \\
Type 2 DM risk score & 0.024 & 0.0089 & 0.007 \\
Body mass index $\left(\mathrm{kg} / \mathrm{m}^{2}\right)$ & 0.0031 & 0.0024 & 0.2 \\
$\sqrt{\text { age onset }} \times$ gender & -0.079 & 0.015 & $1 \mathrm{e}-07$ \\
$\sqrt{\text { age onset }} \times$ duration & -0.0089 & 0.00059 & $2 \mathrm{e}-51$ \\
$\sqrt{\text { age onset }} \times$ Type 1 score & -0.0087 & 0.0058 & 0.1 \\
$\sqrt{\text { age onset }} \times$ Type 2 score & 0.0042 & 0.0059 & 0.5 \\
$\sqrt{\text { age onset }} \times$ BMl & 0.0044 & 0.0017 & 0.008 \\
\hline
\end{tabular}

\section{Associations of age at onset and C-peptide with SNP genotypes}

Genome-wide associations of age at onset and C-peptide levels with SNP genotypes are summarized in Manhattan plots in which regions containing diabetes-associated SNPs (based on those included in the genotypic scores) are highlighted (Figs. 3 and 4). The regression models for age at onset included sex as covariate, and regression models for C-peptide levels included sex, age at onset, duration and age at onset $x$ duration. The only SNP associations with age at onset and C-peptide for which the $p$ values were less than $10^{-7}$ were in the HLA and INS gene regions. The reported association of the PTPN22 $185 \mathrm{~T}$ variant (rs2476601) with C-peptide persistence [22] was confirmed (Table 4). Reported associations of SNPs outside the HLA and INS regions with age at onset [23] or associations of SNPs in the HLA region with C-peptide but not age at onset [6] were not confirmed in this study (Table 4). The SNP rs1983890 near the PFKFB3 gene, previously reported to be associated with latent autoimmune diabetes of adulthood [24], was not associated with age at onset or with detectable C-peptide secretion (Table 4).

\section{Associations of age at onset and C-peptide with locus-specific genotypic risk scores}

In Fig. 5, the effects of locus-specific genotypic scores are displayed by plotting the regression slope for $\mathrm{C}$-peptide (adjusted for gender, age of onset and duration) against

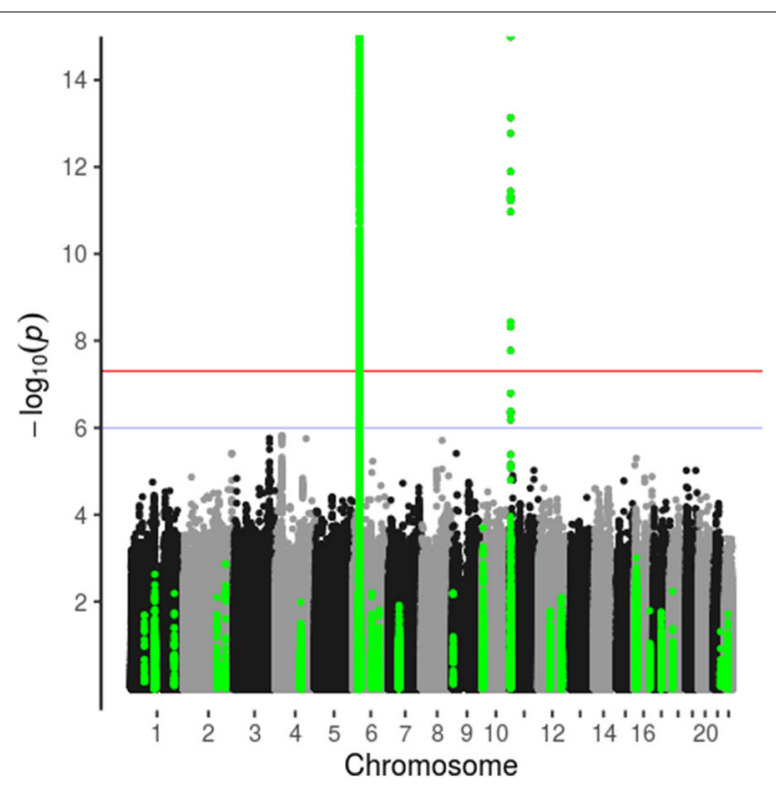

Fig. 3 Manhattan plot of genome-wide association study of age at onset (vertical axis truncated at 15). Type 1 diabetes-associated regions in green 


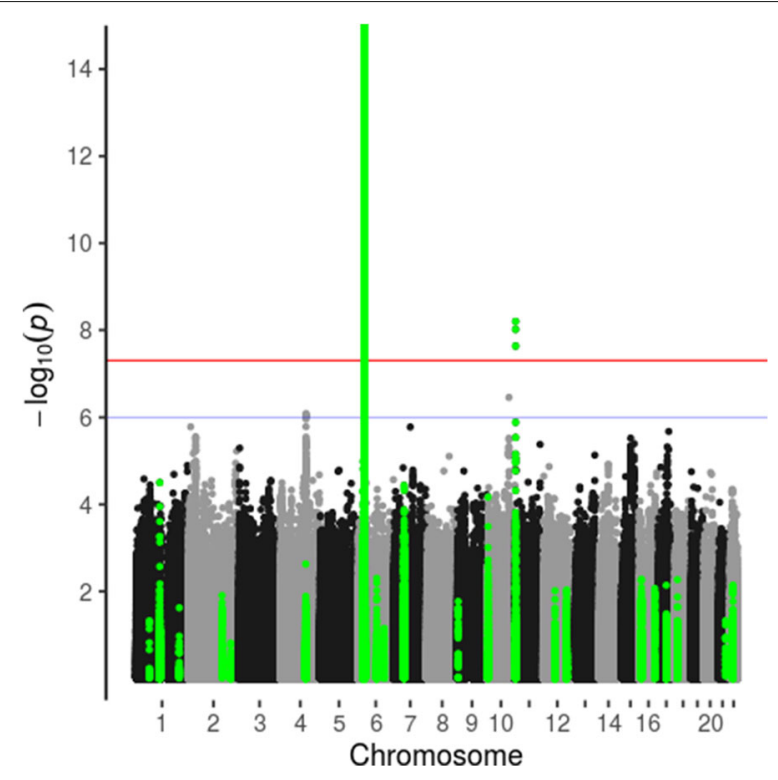

Fig. 4 Manhattan plot of genome-wide association study of C-peptide levels, adjusted for age at onset, duration and predicted age at onset given genotype (vertical axis truncated at 15). Type 1 diabetes-associated regions in green

the regression slope for age at onset. The regression coefficients and $p$ values are given in Additional file 1: Tables 5, 6, 7 and 8. The HLA residual score for type 1 diabetes, calculated by adjusting the HLA region-specific polygenic score for HLA serotype score, represents the residual contribution of the HLA region to risk of type 1 diabetes that is not explained by HLA serotype.

This plot shows that by far the strongest genetic effect on age at onset is that of HLA serotype. Other type 1 diabetes-associated regions that contribute are the INS,

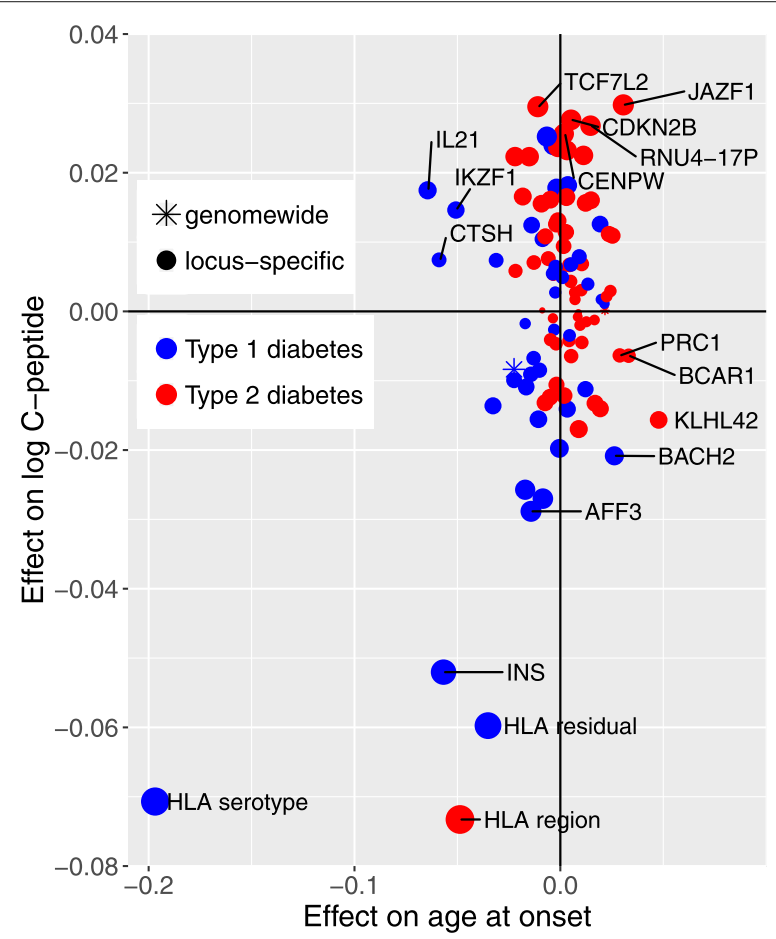

Fig. 5 Locus-specific genotypic scores for type 1 or type 2 diabetes: plot of univariate effects on log 10 C-peptide (adjusted for age at onset and duration) against univariate effect on square root age at onset. Effect sizes are the effect of a change of one standard deviation in each locus-specific score 
Table 4 GWAS results for age at onset and for C-peptide adjusted for sex, age at onset and duration: associations with SNPs previously reported

\begin{tabular}{lllllllll}
\hline Chr & SNP & Position & Alleles & Freq & Score age onset & $p$ value age onset & Score C-peptide & $p$ value C-peptide \\
\hline 1 & rs2476601 & 114377568 & A/G & 0.83 & 9.4 & 0.7 & 160.8 & $3 e-04$ \\
6 & rs72975913 & 128293932 & A/C & 0.85 & -36.9 & 0.1 & -16.9 & 0.7 \\
10 & rs1983890 & 6178614 & T/C & 0.66 & -28.9 & 0.4 & -32.4 & 0.6 \\
6 & rs9260151 & 29911030 & T/C & 0.91 & -39.1 & 0.04 & -100.6 & 0.003 \\
6 & rs1264813 & 29939900 & T/C & 0.91 & 96.6 & $3 e-07$ & 123.3 & $2 e-04$ \\
6 & rs61211515 & 30100975 & C/CT & 0.87 & 79.2 & $3 e-04$ & 107.3 & 0.005 \\
6 & rs3135002 & 32668439 & A/C & 0.96 & -124.2 & $6 e-20$ & -258.3 & $3 e-28$ \\
1 & rs559047 & 238753916 & A/T & 0.77 & -16.9 & 0.6 & -37 & 0.5 \\
\hline
\end{tabular}

Sources for reported association: rs2476601 (C1858T variant in PTPN22) [22], rs72975913 [23], rs1983890 [24], all others [6]

IL21 and CTSH gene regions. Scores for type 2 diabetes were not in general associated with age at onset.

For C-peptide persistence, the strongest effect was that of the HLA score for type 2 diabetes. Several locusspecific scores for type 2 diabetes, including $J A Z F 1$ and $T C F 7 L 2$, are associated with C-peptide persistence. For a few loci, the effects on age at onset and the effects on $\mathrm{C}$ peptide levels appear to be discrepant in direction or size. Thus, the IL21 score for type 1 diabetes was strongly associated with early age at onset but not with low C-peptide level. The KHL42 score for type 2 diabetes was associated with late age at onset but not with high $\mathrm{C}$-peptide levels. The score for the CTSH gene region, in which SNP genotypes were previously reported to be associated with clinical remission at 1 year from diagnosis in children with type 1 diabetes [25], was strongly associated with age at onset but not with $\mathrm{C}$-peptide persistence.

Of the five loci that were common to both types of diabetes, there were four-BMP8A, HLA region, CENPW, $A S C C 2$-for which scores for type 1 and type 2 diabetes were positively correlated in the type 1 Bioresource implying that the same alleles or haplotypes are associated with increased risk of both types of diabetes. For the $B C A R 1 / C T R B 1 / C T R B 2$ region, the scores were negatively correlated $(r=-0.20)$, consistent with studies showing that the G allele of the rs720877 SNP in this region is associated with increased risk of type 1 diabetes but with decreased risk of type 2 diabetes [14, 15, 26].

\section{Discussion}

\section{Frequency of detectable C-peptide secretion}

The high prevalence of detectable $\mathrm{C}$-peptide in this cohort is consistent with most other recent studies [3, 4] that have used highly sensitive assays. Of the 585 patients aged less than 15 years at onset and with at least 35 years duration, $85 \%$ had no $\mathrm{C}$-peptide detectable with this assay. Although detectable C-peptide secretion is common, for those diagnosed in childhood, however, our results do not support the assertion that "the majority of patients with long-duration Type 1 diabetes are insulin microsecretors" [2]. Although we did not use a mixed meal to stimulate Cpeptide secretion, plasma glucose measured on the same samples (dichotomized at $8 \mathrm{mmol} / \mathrm{l}$ ) was not associated with $\mathrm{C}$-peptide level in a regression model. Comparisons of the prevalence of detectable $\mathrm{C}$-peptide secretion between studies are difficult to interpret because these studies differ in distribution of age at onset, gender, criteria for type 1 diabetes and detection limits of the assays used.

If this cross-sectional population-based sample represents the natural history of $\mathrm{C}$-peptide persistence in type 1 diabetes, we can infer that $\mathrm{C}$-peptide falls rapidly within the first 10 years in early-onset cases, but more slowly in late-onset cases, continuing to decline more than ten years after diagnosis. A similar pattern was reported in a European multicentre study [5]. This is consistent with cross-sectional results reported previously for individuals diagnosed after 11 years of age [27]. An unexpected finding was that in late-onset cases, $\mathrm{C}$-peptide levels appear to fall off more rapidly in women than in men.

The clinical picture labelled as latent autoimmune diabetes in adults [28, 29], in which islet antibodies are present but insulin is not required during the first 6 months after diagnosis [30,31], may simply be one end of a spectrum from classic juvenile-onset type 1 diabetes to later-onset cases with slowly progressive loss of betacell function [32, 33]. The absence of auto-antibodies does not necessarily exclude autoimmune pathogenesis of diabetes even in people with high C-peptide levels [34]. However, we have validated our classification of type 1 and possible type 2 diabetes by comparing the mean genotypic risk scores of these categories in the SDRNT1BIO cohort with the distributions of those who can be reliably classified as type 1 or type 2 in the UK Biobank cohort. Further confirmation that we have managed to exclude misdiagnosed cases of type 2 is that age at onset is not associated with genotypic risk score for type 2 in those we have classified as type 1 . A practical implication is that in late-onset 
cases presenting with a clinical picture that is compatible with type 1 diabetes, autoantibody testing might be useful in excluding cases of type 2 diabetes who will not need long-term insulin therapy. We have noted that summary GWAS results for type 1 and type 2 diabetes show at least four risk loci where the same alleles are associated with increased risk of both types, implying some overlap in aetiologies.

\section{Genetic associations with age at onset and C-peptide persistence}

Other studies of genetic associations with age at onset and $\mathrm{C}$-peptide persistence in type 1 diabetes have tested individual SNPs for association as in a conventional GWAS $[6,22,23]$. We have undertaken a GWAS but have also taken a different approach, using publicly available summary GWAS results to compute genotypic risk scores for each diabetes-associated genomic region. Because the prior hypothesis space is far smaller than it would be in a conventional GWAS, this approach makes it possible to detect effects that would be missed in a conventional GWAS of individual SNPs.

As C-peptide persistence depends on age at onset, a first step in understanding the genetic architecture of $\mathrm{C}$ peptide persistence is to study genetic effects on age at onset. Others have reported [35] that average genetic risk scores for type 1 diabetes are lower in late-onset than in early-onset cases of type 1 diabetes. To estimate the relative mixture proportions of type 1 and type 2 on the assumption that the distribution of genotypic risk scores in type 1 diabetes is independent of age at onset [36] is likely to underestimate the proportion of type 1 cases. In contrast to a recent meta-analysis of 15696 cases typed with the ImmunoChip [23] which found strong evidence for association with age at onset in only two genomic regions-the HLA region and the PTPRK / THEMIS region on 6q22.33-we find that several other risk loci for type 1 diabetes are also associated with age at onset. The strongest effect on age at onset of any region outside the $H L A$ region is the $I L 21$ gene region, which has a relatively small effect on risk of type 1 diabetes itself.

Because C-peptide persistence is related to age at onset, to detect specific genetic effects on C-peptide secretion, it is necessary to adjust for age at onset. Although the SNP heritability of residual C-peptide adjusted for gender, age at onset and duration is $26 \%$, genetic risk scores for diabetes explain only an additional $1 \%$ of variance in this adjusted phenotype and most of the remaining genetic and environmental variance remains unexplained. By adjusting the polygenic score for type 1 diabetes in the HLA region for HLA serotype at the HLA$D R B 1$ and $H L A-D Q B 1$ loci, we were able to distinguish the effects of serotype from the effects of other genes in the HLA region. The strongest effects on age at onset (as on type 1 diabetes itself) are from HLA serotype, but for $C$-peptide, the strongest effects are those of variants in the HLA region that are independent of HLA serotype and are associated with increased risk of type 1 and type 2 diabetes. To investigate further the effects of the HLA region on $\mathrm{C}$-peptide persistence that are distinct from the effects of this region on risk of juvenile-onset diabetes, it will be necessary to model the joint effects of HLA genes in case-control comparisons combined with caseonly studies of C-peptide persistence. We note also that some risk loci for type 2 diabetes are associated with Cpeptide persistence but not with age at onset: this implies that they influence the clinical phenotype of late-onset type 1 diabetes even though they do not influence the risk of developing this condition.

\section{Conclusions}

Persistence of $\mathrm{C}$-peptide secretion varies widely in people clinically diagnosed as type 1 diabetes. Known risk loci for diabetes account for only a small proportion of the genetic effects on C-peptide persistence. C-peptide persistence is influenced by variants in the HLA region that are different from those determining risk of early-onset type 1 diabetes. Further exploration of how genetic effects on C-peptide persistence differ from the established genetic effects on islet-cell autoimmunity may provide insights into pathways that could be targeted to limit or reverse the loss of beta cell function in type 1 diabetes.

\section{Additional file}

Additional file 1: Table 5. Regressions of age at onset on regional genotypic scores for type 1 diabetes. Table $\mathbf{6}$. Regressions of age at onset on regional genotypic scores for type 2 diabetes. Table 7. Regressions of $\log$ C-peptide on regional genotypic scores for type 1 diabetes. Table $\mathbf{8}$. Regressions of $\log C$-peptide on regional genotypic scores for type 2 diabetes. (PDF $34 \mathrm{~kb}$ )

\section{Abbreviations}

GAD65: Glutamic acid decarboxylase; GWAS: Genome-wide association study; HLA: Human leukocyte antigen; IA2: Tyrosine phosphorylase-related protein 2; SDRNT1BIO or T1Bio: Scottish Diabetes Research Network Type 1 Bioresource; WHO: World Health Organization; ZnT8: Zinc transporter 8

\section{Acknowledgements}

We thank the fieldworkers, supervisors and collaborators from the NHS health boards across Scotland for facilitating this study.

\section{Authors' contributions}

$\mathrm{HC}$ and PM provided substantial contributions to the conception or design and drafting of the manuscript. CP, TM, SO-G, SR, JM, AP, JC, RL, JP, ST, AC, SM and $\mathrm{HC}$ provided substantial contributions to the data acquisition. PM, HC, AC, MC, SM, LB, SR, TM and MS provided substantial contributions to the data analysis or interpretation. All authors contributed to revising the manuscript critically for important intellectual content and approved the final manuscript. The funding agencies had no part in writing or reviewing the manuscript.

\section{Funding}

The establishment of the SDRN Type 1 Bioresource was supported by the Chief Scientist Office of the Scottish Government Health and Social Care Directorates 
[ETM/47] by Diabetes UK [10/0004010] and by in-kind contributions from the Scottish Diabetes Research Network to facilitate recruitment. The genotyping was supported by the Juvenile Diabetes Research Fund [17-2013-7]. The funding bodies had no role in the design, conduct or reporting of this study.

\section{Availability of data and materials}

The aggregate-level data objects together with the Rmarkdown script that generated this manuscript are available from the corresponding author upon request. In accordance with governance requirements, a data access committee oversees applications for access to individual-level data as described elsewhere [8].

\section{Ethics approval and consent to participate}

The study was carried out in accordance with the ethical principles in the Declaration of Helsinki and was approved by the Tayside Research Ethics Committee (reference 10/S1402/43). Informed consent was obtained from all participants.

\section{Consent for publication}

Not applicable.

\section{Competing interests}

$\mathrm{HC}$ receives research support and honoraria and is a member of advisory panels or speaker's bureaus for Sanofi Aventis, Regeneron, Novartis, Novo-Nordisk and Eli Lilly. HC receives or has recently received non-binding research support from Pfizer, AstraZeneca and Novo-Nordisk. HC holds shares in Roche and Bayer. RL receives personal fees and non-financial support from Novo Nordisk, personal fees from Eli Lilly Ltd and non-financial support from Merck Sharp and Dohme. JP receives personal fees from Novo Nordisk, non-financial support from Merck (Germany) and Itamar Medical, personal fees from Lilly, ACI Clinical, Pfizer, Astra Zeneca and grants from Janssen. The other authors declare that they have no competing interests.

\section{Author details}

${ }^{1}$ Usher Institute of Population Health and Informatics, University of Edinburgh, Old Medical School, Teviot Place, Edinburgh EH8 9AG, UK. Institute of Genetics and Molecular Medicine, University of Edinburgh, Western General Hospital Campus, Crewe Road, Edinburgh, UK. ${ }^{3}$ Institute of Cardiovascular and Medical Sciences, University of Glasgow, Glasgow, UK. ${ }^{4}$ Royal Infirmary of Edinburgh, Edinburgh, UK. ${ }^{5}$ Metabolic Unit, Western General Hospital, Edinburgh, UK. ${ }^{6}$ Diabetes Centre, Victoria Hospital, Kirkaldy, UK. ${ }^{7}$ David Matthews Diabetes Centre, Monklands Hospital, Airdrie, UK. ${ }^{8}$ Center for Public Health Genomics, University of Virginia, Charlottesville, USA. ${ }^{9}$ Medical School, University of Exeter, Exeter, UK. ${ }^{10}$ Medical School, University of Dundee, Dundee, UK. ${ }^{11}$ Glasgow Caledonian University, Glasgow, UK. ${ }^{12}$ NHS Highland Diabetes Centre, Inverness, UK.

\section{Received: 13 March 2019 Accepted: 15 July 2019}

Published online: 23 August 2019

\section{References}

1. Madsbad S, Faber OK, Binder C, McNair P, Christiansen C, Transbøl I. Prevalence of residual beta-cell function in insulin-dependent diabetics in relation to age at onset and duration of diabetes. Diabetes. 1978;27 Suppl 1:262-4.

2. Oram RA, Jones AG, Besser REJ, Knight BA, Shields BM, Brown RJ, et al. The majority of patients with long-duration type 1 diabetes are insulin microsecretors and have functioning beta cells. Diabetologia. 2014;57: 187-91.

3. Davis AK, DuBose SN, Haller MJ, Miller KM, DiMeglio LA, Bethin KE, et al. Prevalence of detectable C-Peptide according to age at diagnosis and duration of type 1 diabetes. Diabetes Care. 2015;38:476-81.

4. Li X, Cheng J, Zhou Z. Revisiting multiple models of progression of beta-cell loss of function in type 1 diabetes: significance for prevention and cure. J Diabetes. 2016;8:460-9.

5. Barker A, Lauria A, Schloot N, Hosszufalusi N, Ludvigsson J, Mathieu C, et al. Age-dependent decline of $\beta$-cell function in type 1 diabetes after diagnosis: A multi-centre longitudinal study. Diabetes Obes Metab. 2014;16:262-7.
6. Roshandel D, Gubitosi-Klug R, Bull SB, Canty AJ, Pezzolesi MG, King GL, et al. Meta-genome-wide association studies identify a locus on chromosome 1 and multiple variants in the MHC region for serum C-peptide in type 1 diabetes. Diabetologia. 2018;61:1098-111.

7. Livingstone SJ, Levin D, Looker HC, Lindsay RS, Wild SH, Joss N, et al. Estimated life expectancy in a Scottish cohort with type 1 diabetes, 2008-2010. JAMA. 2015;313:37-44.

8. AkbarT, McGurnaghan S, Palmer CNA, Livingstone SJ, Petrie J, Chalmers J, et al. Cohort Profile: Scottish Diabetes Research Network Type 1 Bioresource Study (SDRNT1BIO). Int J Epidemiol. 2017;46:796-796i.

9. Hope SV, Knight BA, Shields BM, Hattersley AT, McDonald TJ, Jones AG. Random non-fasting C-peptide: bringing robust assessment of endogenous insulin secretion to the clinic. Diabet Med. 2016;33:1554-8.

10. McDonald TJ, Knight BA, Shields BM, Bowman P, Salzmann MB, Hattersley AT. Stability and reproducibility of a single-sample urinary C-peptide/creatinine ratio and its correlation with 24-h urinary C-peptide. Clin Chem. 2009;55:2035-9.

11. Schlosser M, Mueller PW, Achenbach P, Lampasona V, Bingley PJ. Participating Laboratories. Diabetes Antibody Standardization Program: first evaluation of assays for autoantibodies to IA-2 $\beta$. Diabetes Care. 2011;34:2410-2.

12. Loh P-R, Palamara PF, Price AL. Fast and accurate long-range phasing in a UK Biobank cohort. Nat Genet. 2016;48:811-6.

13. Spiliopoulou A, Colombo M, Plant D, Nair N, Cui J, Coenen MJ, et al. Association of response to TNF inhibitors in rheumatoid arthritis with quantitative trait loci for CD40 and CD39. Annals Rheum Dis. 2019.

14. Barrett JC, Clayton DG, Concannon P, Akolkar B, Cooper JD, Erlich HA, et al. Genome-wide association study and meta-analysis find that over 40 loci affect risk of type 1 diabetes. Nat Genet. 2009;41:703-7.

15. Morris $A P$, Voight $B F$, Teslovich $T M$, Ferreira $T$, Segrè AV, Steinthorsdottir $V$, et al. Large-scale association analysis provides insights into the genetic architecture and pathophysiology of type 2 diabetes. Nat Genet. 2012;44: 981-90.

16. Onengut-Gumuscu S, Chen W-M, Burren O, Cooper NJ, Quinlan AR, Mychaleckyj JC, et al. Fine mapping of type 1 diabetes susceptibility loci and evidence for colocalization of causal variants with lymphoid gene enhancers. Nat Genet. 2015;47:381-6.

17. Zheng X, Shen J, Cox C, Wakefield JC, Ehm MG, Nelson MR, et al. HIBAG - HLA genotype imputation with attribute bagging. Pharmacogenomics J. 2014;14:192-200.

18. Gourraud P-A, Khankhanian P, Cereb N, Yang SY, Feolo M, Maiers M, et al. HLA Diversity in the 1000 Genomes Dataset. PLoS ONE. 20149.

19. Oram RA, Patel K, Hill A, Shields B, McDonald TJ, Jones A, et al. A type 1 diabetes genetic risk score can aid discrimination between type 1 and type 2 diabetes in young adults. Diabetes Care. 2016;39:337-44.

20. Chen H, Wang C, Conomos MP, Stilp AM, Li Z, Sofer T, et al. Control for population structure and relatedness for binary traits in genetic association studies via logistic mixed models. Am J Human Genet. 2016;98:653-66.

21. Kuhtreiber WM, Washer SLL, Hsu E, Zhao M, Reinhold P, Burger D, et al. Low levels of C-peptide have clinical significance for established type 1 diabetes. Diabet Med A J B Diabet Assoc. 2015;32:1346-53.

22. Petrone A, Spoletini M, Zampetti S, Capizzi M, Zavarella S, Osborn J, et al. The PTPN22 1858T gene variant in type 1 diabetes is associated with reduced residual beta-cell function and worse metabolic control. Diabetes Care. 2008;31:1214-8.

23. Inshaw JRJ, Walker NM, Wallace C, Bottolo L, Todd JA. The chromosome 6q22.33 region is associated with age at diagnosis of type 1 diabetes and disease risk in those diagnosed under 5 years of age. Diabetologia. 2017;61:147-57.

24. Cousminer DL, Ahlqvist E, Mishra R, Andersen MK, Chesi A, Hawa MI, et al. First genome-wide association study of latent autoimmune diabetes in adults reveals novel insights linking immune and metabolic diabetes. Diabetes Care. 2018;41:2396-403.

25. Fløyel T, Brorsson C, Nielsen LB, Miani M, Bang-Berthelsen CH, Friedrichsen $\mathrm{M}$, et al. CTSH regulates $\beta$-cell function and disease progression in newly diagnosed type 1 diabetes patients. Proc Natl Acad Sci U S A. 2014;111:10305-10.

26. 't Hart LM, Fritsche A, Nijpels G, van Leeuwen N, Donnelly LA, Dekker $J M$, et al. The CTRB1/2 locus affects diabetes susceptibility and treatment via the incretin pathway. Diabetes. 2013;62:3275-81. 
27. Shields BM, McDonald TJ, Oram R, Hill A, Hudson M, Leete P, et al. C-Peptide decline in type 1 diabetes has two phases: an initial exponential fall and a subsequent stable phase. Diabetes Care. 2018:41:1486-92.

28. Zimmet PZ, Tuomi T, Mackay IR, Rowley MJ, Knowles W, Cohen M, et al. Latent autoimmune diabetes mellitus in adults (LADA): the role of antibodies to glutamic acid decarboxylase in diagnosis and prediction of insulin dependency. Diabet Med. 1994;11:299-303.

29. Mishra R, Hodge KM, Cousminer DL, Leslie RD, Grant SFA. A global perspective of latent autoimmune diabetes in adults. Trends Endocrinol Metab. 2018;29:638-50.

30. Groop LC, Bottazzo GF, Doniach D. Islet cell antibodies identify latent type I diabetes in patients aged 35-75 years at diagnosis. Diabetes. 1986;35:237-41

31. Prior MJ, Prout T, Miller D, Ewart R, Kumar D. C-peptide and the classification of diabetes mellitus patients in the Early Treatment Diabetic Retinopathy Study. Report number 6. The ETDRS Research Group. Annals Epidemiol. 1993;3:9-17.

32. Leslie RDG, Kolb H, Schloot NC, Buzzetti R, Mauricio D, De Leiva A, et al. Diabetes classification: grey zones, sound and smoke: Action LADA 1. Diabetes Metab Res Rev. 2008;24:511-9.

33. Tuomi T, Santoro N, Caprio S, Cai M, Weng J, Groop L. The many faces of diabetes: a disease with increasing heterogeneity. Lancet (London, England). 2014;383:1084-94.

34. Brooks-Worrell BM, lyer D, Coraza I, Hampe CS, Nalini R, Ozer K, et al Islet-specific T-cell responses and proinflammatory monocytes define subtypes of autoantibody-negative ketosis-prone diabetes. Diabetes Care. 2013;36:4098-103.

35. Mishra R, Chesi A, Cousminer DL, Hawa MI, Bradfield JP, Hodge KM, et al. Relative contribution of type 1 and type 2 diabetes loci to the genetic etiology of adult-onset, non-insulin-requiring autoimmune diabetes. BMC Med. 2017;15:88.

36. Thomas NJ, Jones SE, Weedon MN, Shields BM, Oram RA, Hattersley AT. Frequency and phenotype of type 1 diabetes in the first six decades of life: a cross-sectional, genetically stratified survival analysis from UK Biobank. Lancet Diabetes Endocrinol. 2018;6:122-9.

\section{Publisher's Note}

Springer Nature remains neutral with regard to jurisdictional claims in published maps and institutional affiliations.

Ready to submit your research? Choose BMC and benefit from:

- fast, convenient online submission

- thorough peer review by experienced researchers in your field

- rapid publication on acceptance

- support for research data, including large and complex data types

- gold Open Access which fosters wider collaboration and increased citations

- maximum visibility for your research: over $100 \mathrm{M}$ website views per year

At BMC, research is always in progress.

Learn more biomedcentral.com/submissions 